\title{
ABSORÇÃO E TRANSLOCAÇÃO DE SILÍCIO EM MUDAS DE EUCALIPTO CULTIVADAS EM LATOSSOLO E CAMBISSOLO ${ }^{1}$
}

\author{
RUY CARVALHO ${ }^{2}$ \\ ANTONIO EDUARDO FURTINI NETO ${ }^{3}$ \\ NILTON CURI ${ }^{4}$ \\ ÁLVARO VILELA DE RESENDE ${ }^{5}$
}

\begin{abstract}
RESUMO - Com o objetivo de estudar a absorção e a translocação de Si em mudas de Eucalyptus grandis W. Hill. Ex Maiden cultivadas em amostras de Latossolo Vermelho distrófico e de Cambissolo Háplico Tb distrófico, foram realizados dois experimentos em casa-devegetação. Cada solo foi submetido a três incubações seqüenciais para a aplicação dos tratamentos: a) Ca$\mathrm{CO}_{3}+\mathrm{MgCO}_{3}$ para manter o $\mathrm{pH}$ em torno de 6,$0 ; \mathrm{b}$ ) fertilização básica com macro e micronutrientes; c) seis doses de $\mathrm{Si}\left(\mathrm{CaSiO}_{3}\right)$. As plantas foram cultivadas em vasos contendo $2,7 \mathrm{dm}^{3}$ de solo. O teor de $\mathrm{Si}$ solúvel em água no solo e o conteúdo de Si nas raízes e parte aérea das plantas foram avaliados aos 60, 90 e 120 dias do
\end{abstract}

\begin{abstract}
transplantio, ajustando-se superfícies de resposta dessas variáveis às doses de $\mathrm{Si}$ e às épocas. Determinaram-se os índices de eficiência de absorção e de utilização do $\mathrm{Si}$ pelas plantas e a porcentagem de $\mathrm{Si}$ translocado. $\mathrm{Na}$ fase inicial de crescimento (60 dias), as mudas absorveram muito pouco $\mathrm{Si}$, e a eficiência de translocação foi máxima aos 60 dias, ficando a maior parte do elemento retida nas raízes a partir dessa época. O teor de Si solúvel em água no solo reduziu-se com o tempo, mas a absorção do elemento pelo eucalipto não foi responsável pelo fato. Os dados obtidos evidenciaram que o eucalipto não é uma planta acumuladora de $\mathrm{Si}$, embora seja responsiva ao mesmo.
\end{abstract}

TERMOS PARA INDEXAÇÃO: Eucalyptus grandis, acúmulo de silício, espécie florestal.

\section{SILICON UPTAKE AND TRANSLOCATION BY EUCALYPTUS SEEDLINGS CULTIVATED IN LATOSOL (OXISOL) AND CAMBISOL (INCEPTISOL)}

\begin{abstract}
With the objective of studying the absorption and the translocation of $\mathrm{Si}$ by seedlings of Eucalyptus grandis W. Hill. Ex Maiden cultivated in samples of a dystrophic Red Latosol (Oxisol) and of a dystrophic Haplic Cambisol (Inceptisol), two experiments were carried out under greenhouse conditions. Each soil was submitted to three sequencial incubations for the application of the treatments: a) $\mathrm{CaCO}_{3}+\mathrm{MgCO}_{3}$ to maintain the $\mathrm{pH}$ around $6.0 ; \mathrm{b}$ ) basic fertilization with macro and micronutrients; c) six doses of $\mathrm{Si}\left(\mathrm{CaSiO}_{3}\right)$. The plants were grown in pots containing $2.7 \mathrm{dm}^{3}$ of soil. The water-soluble $\mathrm{Si}$ in the soil and the $\mathrm{Si}$ content in the plant roots and shoot were evaluated at 60, 90, and 120 days
\end{abstract}

after seedlings transplanting. Response surfaces for those variables were adjusted as a function of the $\mathrm{Si}$ doses and evaluation epoch. The indices of uptake and use efficiency of the Si by plants and the percentage of translocated $\mathrm{Si}$ were determined. In the initial growth phase (60 days), the seedlings absorbed very little amounts of $\mathrm{Si}$. The translocation efficiency was highest at 60 days, being most of the element retained in the roots after that time. The amount of water-soluble $\mathrm{Si}$ in the soil was reduced with the time, but the uptake of the element by the Eucalyptus was not related to this fact. The obtained data evidenced the Eucalyptus is a non-accumulator plant, although responsive to $\mathrm{Si}$.

INDEX TERMS: Eucalyptus grandis, silicon accumulation, forest species.

1. Trabalho financiado pela FAPEMIG.

2. Engenheiro Agrônomo, D.Sc., Professor Titular, Departamento de Química, UNIVERSIDADE FEDERAL DE LAVRAS/UFLA, Caixa Postal 37, 37200-000 - Lavras, MG. ruy@ufla.br

3. Engenheiro Agrônomo, D.Sc., Professor Adjunto, Departamento de Ciência do Solo/UFLA. Bolsista do CNPq. afurtini@ufla.br

4. Engenheiro Agrônomo, PhD., Professor Titular, Departamento de Ciência do Solo/UFLA. Bolsista do CNPq. niltcuri@ufla.br

5. Engenheiro Agrônomo, M.Sc., Pesquisador, Embrapa Cerrados, Caixa Postal 08223, 73301-970, Planaltina, DF. álvaro@cpac.embrapa.br 


\section{INTRODUÇÃO}

O composto de silício presente na fase líquida do solo, na faixa de pH de 4 a 9, é o monômero $\mathrm{H}_{4} \mathrm{SiO}_{4}$ (McKeague \& Cline, 1963a), forma pela qual o elemento é também absorvido pelas plantas (Marschner, 1995). De acordo com esse autor, as diferentes espécies vegetais variam grandemente em sua capacidade de absorver e acumular $\mathrm{Si}$ nos tecidos, podendo, em função dos percentuais de $\mathrm{SiO}_{2}$ na matéria seca da parte aérea, serem classificadas como: a) plantas acumuladoras, que incluem muitas gramíneas como o arroz, contendo de 10 a $15 \mathrm{dag} \mathrm{kg}^{-1}$ de $\mathrm{SiO}_{2}$; b) intermediárias, com teores de $\mathrm{SiO}_{2}$ variando de 1 a $5 \mathrm{dag} \mathrm{kg}^{-1}$ (cereais, cana-deaçúcar e poucas dicotiledôneas); c) plantas nãoacumuladoras, incluindo a maioria das dicotiledôneas, apresentando $<0,5 \mathrm{dag} \mathrm{kg}^{-1}$ de $\mathrm{SiO}_{2}$.

De modo geral, nas monocotiledôneas (arroz, cana-de-açúcar, trigo, cevada, centeio, milho, aveia, sorgo, milheto, etc.), o destino da maior parte do Si transportado das raízes pela corrente transpiratória é acumulado (precipitado) como $\mathrm{SiO}_{2}$ em órgãos da parte aérea, tornando-se imóvel e, portanto, não sendo redistribuído (Jones \& Handreck, 1967). Para essas espécies, embora não seja considerado um elemento essencial, o Si desempenha um papel importante para o crescimento e a produtividade, havendo sugestões inclusive de o mesmo possuir funções metabólicas ainda não muito bem elucidadas, como na produção de lignina, indução de deficiência de Zn e formação de folhas (Emadian \& Newton, 1989; Korndörfer \& Datnoff, 1995; Belanger et al., 1995).

Independentemente do vegetal estudado, parece que a deposição do elemento nas raízes está relacionada com a conservação da água necessária ao vigor dos tecidos durante os períodos de estresse hídrico (Sangster, 1978). A própria química do $\mathrm{Si}$ reforça o papel do $\mathrm{SiO}_{2}$ como amenizante do estresse de água. Possuidor de geometria tetraédrica como o carbono (Joly, 1966), o Si ${ }^{4+}$ presente no $\mathrm{SiO}_{2}$ estabiliza-se com quatro ligações químicas de igual tamanho, sendo comum, por isso, a representação do ácido silícico, $\mathrm{H}_{4} \mathrm{SiO}_{4}$, na forma $\mathrm{Si}(\mathrm{OH})_{4}$. Para chegar a essa estrutura estável, o $\mathrm{SiO}_{2}$ combina-se facilmente com moléculas de água, de acordo com a reação $\mathrm{SiO}_{2}+2 \mathrm{H}_{2} \mathrm{O} \leftrightarrow \mathrm{H}_{4} \mathrm{SiO}_{4}$. Assim, as moléculas de $\mathrm{H}_{4} \mathrm{SiO}_{4}$ poderiam ser consideradas "reservatórios de água" em períodos de estresse hídrico.

Depósitos radiculares de $\mathrm{Si}$ foram detectados principalmente em dicotiledôneas, nas quais o teor de $\mathrm{SiO}_{2}$ nas raízes é relativamente alto em relação à parte aérea (Jones \& Handreck, 1967). Tal deposição de Si foi observada em espécies como pepino (Miyake \& Takahashi, 1983), soja (Miyake \& Takahashi, 1985) e tomate (Okuda \& Takahashi, 1964).

Existem muitas informações disponíveis sobre o comportamento do Si em plantas, com maior ênfase no crescimento e produtividade de gramíneas, o mesmo valendo para legumes e cereais de maior importância econômica. Contudo, poucos esforços têm sido dedicados às espécies arbóreas, como é o caso do eucalipto, amplamente difundido nos reflorestamentos pelo Brasil. Dessa forma, com este trabalho objetivou-se estudar a absorção e a translocação de silício em mudas de $E u$ calyptus grandis W. Hill. Ex Maiden cultivadas sob doses crescentes do elemento em Latossolo e Cambissolo.

\section{MATERIAL E MÉTODOS}

Dois experimentos foram conduzidos em casade-vegetação do Departamento de Ciência do Solo da Universidade Federal de Lavras, Lavras (MG). Utilizaram-se amostras de solo da camada superficial de um Latossolo Vermelho distrófico (LVd) sob cerrado e de um Cambissolo Háplico Tb distrófico (CXbd) sob campo cerrado, cujos atributos químicos, físicos e mineralógicos são apresentados no Quadro 1. A unidade experimental constou de um vaso plástico contendo $2,7 \mathrm{dm}^{3}$ de solo passado em peneira de $2 \mathrm{~mm}$ de malha, no qual foram conduzidas duas plantas de Eucalyptus grandis.

Previamente ao transplantio das mudas, para obter-se o efeito dos tratamentos, todos os vasos foram submetidos a três incubações seqüenciais de 30 dias cada uma, sob condição de umidade equivalente a $60 \%$ do volume total de poros - VTP (Freire et al., 1980): a) uma primeira incubação com uma mistura de Ca$\mathrm{CO}_{3}+\mathrm{MgCO}_{3}$ na relação de mols de $\mathrm{Ca}: \mathrm{Mg}$ igual a 4:1, visando a atingir $\mathrm{pH}$ em torno de 6,0 ; b) a segunda incubação ocorreu após a aplicação de uma fertilização básica com $80 \mathrm{mg} \mathrm{N} ; 150 \mathrm{mg} \mathrm{K} ; 40 \mathrm{mg} \mathrm{S}$; 0,81 mg B; 0,15 mg Mo; 5,0 mg Zn; 3,6 mg Mn; 1,3 mg Cu e 1,6 $\mathrm{mg} \mathrm{Fe}$ por $\mathrm{dm}^{3}$, além de 225 e $150 \mathrm{mg} \mathrm{P} \mathrm{dm}^{-3}$ para o Latossolo e Cambissolo, respectivamente, doses essas deduzidas da Isoterma de Langmuir, suficientes para manter $0,2 \mathrm{mg} \mathrm{P} \mathrm{L}^{-1}$ na fase líquida do solo; c) para a terceira incubação, adicionou-se o $\mathrm{Si}$ em seis doses da fonte $\mathrm{CaSiO}_{3}$, as quais foram $0,112,170,225,280 \mathrm{e}$ $340 \mathrm{mg} \mathrm{dm}^{-3}$ de Si para o Latossolo e 0, 75, 112, 150, 187 e $225 \mathrm{mg} \mathrm{dm}^{-3}$ de Si para o Cambissolo. Reagentes p.a. constituíram as fontes de todos os produtos aplicados aos solos para as incubações. 
QUADRO 1 - Atributos químicos, físicos e mineralógicos dos solos utilizados nos experimentos.

\begin{tabular}{|c|c|c|}
\hline \multirow{2}{*}{ Atributos } & \multicolumn{2}{|c|}{ Solos } \\
\hline & Latossolo & Cambissolo \\
\hline pH em água & 5,0 & 5,2 \\
\hline $\mathrm{P}_{\text {Mehlich-1 }}\left(\mathrm{mg} \mathrm{dm}^{-3}\right)$ & 1 & 1 \\
\hline $\mathrm{P}_{\text {Resina }}\left(\mathrm{mg} \mathrm{dm}{ }^{-3}\right)$ & 2 & 2 \\
\hline $\mathrm{K}\left(\mathrm{mg} \mathrm{dm}^{-3}\right)$ & 32 & 22 \\
\hline $\mathrm{Ca}\left(\mathrm{cmol}_{\mathrm{c}} \mathrm{dm}^{-3}\right)$ & 0,7 & 0,5 \\
\hline $\operatorname{Mg}\left(\mathrm{cmol}_{\mathrm{c}} \mathrm{dm}^{-3}\right)$ & 0,2 & 0,2 \\
\hline $\mathrm{Al}\left(\mathrm{cmol}_{\mathrm{c}} \mathrm{dm}^{-3}\right)$ & 0,3 & 0,7 \\
\hline $\mathrm{H}+\mathrm{Al}\left(\mathrm{cmol}_{\mathrm{c}} \mathrm{dm}^{-3}\right)$ & 6,3 & 6,3 \\
\hline $\mathrm{S}\left(\mathrm{cmol}_{\mathrm{c}} \mathrm{dm}^{-3}\right)$ & 1,0 & 0,7 \\
\hline $\mathrm{t}\left(\mathrm{cmol}_{\mathrm{c}} \mathrm{dm}^{-3}\right)$ & 1,3 & 1,4 \\
\hline $\mathrm{T}\left(\mathrm{cmol}_{\mathrm{c}} \mathrm{dm}^{-3}\right)$ & 7,6 & 6,8 \\
\hline $\mathrm{m}(\%)$ & 20 & 53 \\
\hline $\mathrm{V}(\%)$ & 16 & 10 \\
\hline Matéria orgânica $\left(\mathrm{g} \mathrm{kg}^{-1}\right)$ & 34 & 23 \\
\hline Areia $\left(\mathrm{g} \mathrm{kg}^{-1}\right)$ & 160 & 100 \\
\hline Silte $\left(\mathrm{g} \mathrm{kg}^{-1}\right)$ & 130 & 220 \\
\hline Argila $\left(\mathrm{g} \mathrm{kg}^{-1}\right)$ & 710 & 680 \\
\hline $\mathrm{Fe}_{2} \mathrm{O}_{3} \mathrm{~d}\left(\mathrm{~g} \mathrm{~kg}^{-1}\right)^{1 /}$ & 100 & 80 \\
\hline $\mathrm{Fe}_{\mathrm{o}}\left(\mathrm{g} \mathrm{kg}^{-1}\right)^{2 /}$ & 0,17 & 0,20 \\
\hline $\mathrm{SiO}_{2}\left(\mathrm{~g} \mathrm{~kg}^{-1}\right)^{3 /}$ & 138 & 280 \\
\hline $\mathrm{Al}_{2} \mathrm{O}_{3}\left(\mathrm{~g} \mathrm{~kg}^{-1}\right)^{3 /}$ & 320 & 295 \\
\hline $\mathrm{Fe}_{2} \mathrm{O}_{3}\left(\mathrm{~g} \mathrm{~kg}^{-1}\right)^{3 /}$ & 158 & 160 \\
\hline $\mathrm{TiO}_{2}\left(\mathrm{~g} \mathrm{~kg}^{-1}\right)^{3 /}$ & 21 & 20 \\
\hline $\mathrm{P}_{2} \mathrm{O}_{5}\left(\mathrm{~g} \mathrm{~kg}^{-1}\right)^{3 /}$ & 0,46 & 0,27 \\
\hline CMAF $\left(\mathrm{mg} \mathrm{kg}^{-1}\right)$ & 1666 & 1380 \\
\hline $\mathrm{Ct}\left(\mathrm{g} \mathrm{kg}^{-1}\right)$ & 120 & 280 \\
\hline $\mathrm{Gb}\left(\mathrm{g} \mathrm{kg}^{-1}\right)$ & 290 & 77 \\
\hline $\mathrm{Gt} /(\mathrm{Gt}+\mathrm{Hm})$ & 0,75 & 0,83 \\
\hline Si solúvel em água $\left(\mathrm{mg} \mathrm{dm}^{-3}\right)$ & 29 & 17 \\
\hline
\end{tabular}

${ }^{1 /} \mathrm{Fe}_{2} \mathrm{O}_{3 \mathrm{~d}}=$ ferro ditionito; ${ }^{2 /} \mathrm{Fe}_{0}=$ ferro oxalato; ${ }^{3 /}$ óxidos do ataque sulfúrico; $\mathrm{Ct}=$ caulinita; $\mathrm{Gb}=$ gibbsita; Hm=hematita; Gt=goethita; CMAF=capacidade máxima de adsorção de fósforo. 
Em cada solo, uma das doses de Si foi numericamente igual à quantidade de $\mathrm{P}$ fornecida. As demais doses foram definidas num intervalo que correspondeu, nos seus extremos, a 0,5 e 1,5 vezes a dose de P. Como a fonte de $\mathrm{Si}$ possui o $\mathrm{Ca}^{2+}$ como cátion acompanhante, procedeu-se ao nivelamento do fornecimento do nutriente com $\mathrm{CaCO}_{3}$ p.a. nos demais tratamentos em relação ao $\mathrm{Ca}^{2+}$ suprido com a maior dose de Si. Esse procedimento permitiu também a equiparação dos valores de pH após a aplicação dos tratamentos.

Os ensaios tiveram um delineamento experimental inteiramente casualizado com quatro repetições, arranjado num fatorial $6 \times 3$ constituído pelas 6 doses de $\mathrm{Si}$ e 3 períodos de crescimento do eucalipto $(60,90$ e 120 dias após o transplantio), perfazendo 72 vasos por ensaio envolvendo cada solo.

O transplantio das mudas produzidas em areia lavada para os vasos foi feito quando essas apresentavam quatro pares de folhas. Após os períodos de crescimento pré-estabelecidos, as plantas foram colhidas, separadas em parte aérea e raízes, secas em estufa $\left(70{ }^{\circ} \mathrm{C}\right)$, pesadas e moídas, sendo o Si nos tecidos vegetais analisado segundo o método proposto por Gallo \& Furlani (1978). A determinação do Si solúvel nos solos em cada época foi executada conforme McKeague \& Cline (1963b).

Foram ajustadas superfícies de resposta nas quais as variáveis Si na matéria seca de raízes e da parte aérea das plantas e teor de Si solúvel no solo foram plotadas em função das doses de Si aplicadas e das épocas do monitoramento experimental. As discussões sobre a absorção basearam-se nos índices de eficiência de absorção e de utilização de $\mathrm{Si}$ pelas plantas calculados, respectivamente, conforme Marschner (1995) e Siddiqi \& Glass (1981):

Eficiência de Absorção = [Conteúdo de Si na planta, mg] / [Matéria seca de raízes, $g$ ]

Eficiência de Utilização = [Matéria seca da planta, $g]^{2}$ / [Conteúdo de Si na planta, mg]

Embora a eficiência de utilização seja uma metodologia aplicada em estudos de nutrição mineral de elementos essenciais às plantas, decidiu-se pelo seu uso no presente trabalho com silício.

A translocação de Si foi avaliada com base na relação conteúdo de Si na parte aérea/conteúdo de Si nas raízes e na porcentagem de Si translocado.

\section{RESULTADOS E DISCUSSÃO}

As respostas de crescimento vegetativo do eucalipto em função dos tratamentos utilizados no presente trabalho foram discutidas em Carvalho et al. (2000, 2001), não sendo aqui abordadas.

\section{Silício solúvel no solo e conteúdo de silício nas plantas}

Os teores de Si solúvel em água $\left(\mathrm{H}_{4} \mathrm{SiO}_{4}\right)$ no Latossolo e Cambissolo, bem como os respectivos conteúdos de Si na matéria seca da parte aérea (MSPA) das plantas, são apresentados na Figura 1.

Os sinais, positivo para o termo "Si" e negativo para o termo "E" no modelo de resposta (Figuras 1a e 1b) significam, respectivamente, que o teor de Si solúvel em água (TSi) aumentou, respondendo ao incremento nas doses de $\mathrm{Si}$, e diminuiu em função do tempo. Nos dois casos, os pontos de mínimo Si nos solos, obtidos por derivação dos polinômios, ocorreram aos 96 e 94 dias para o Latossolo e Cambissolo; a partir dessas duas épocas, o TSi voltou a ser incrementado, conforme o termo quadrático $\left(\mathrm{E}^{2}\right)$ com pequena magnitude. Como houve redução do TSi com o tempo, apesar das doses crescentes, parece razoável admitir três possíveis explicações para o fato: adsorção do $\mathrm{Si}$ às superfícies oxídicas da fração argila; insolubilização do elemento por precipitação; e/ou absorção pelas plantas.

Analisando-se as Figuras 1c e 1d, verifica-se que o conteúdo de Si na MSPA (CSi) das plantas cultivadas em ambos os solos aumenta de forma linear com as doses do elemento (sinal positivo do termo "Si" nos polinômios de resposta). Para o experimento com o Latossolo, o CSi apresenta um ponto de mínimo (Figura 1c), ao passo que no Cambissolo ocorre um ponto de máximo CSi (Figura 1d) em função das épocas de avaliação, evidenciando comportamento distinto do eucalipto nos solos estudados.

$\mathrm{O}$ maior acúmulo de $\mathrm{Si}$ nas plantas que cresceram no Latossolo pode ser, em parte, explicado pela presença de menores teores de silte nesse solo (Quadro 1), oferecendo menor impedimento físico à penetração do sistema radicular no vaso de cultivo (Resende et al., 1997), conforme observações feitas durante a condução dos experimentos, o que permitiu maior crescimento das plantas, acompanhado de incremento na absorção do elemento.

Pode-se inferir que, em pequena escala, o eucalipto respondeu em termos de quantidade absorvida aos incrementos de Si no solo; entretanto, não se pode afirmar que a redução no TSi dos dois solos ocorreu de forma tão evidente em razão unicamente da absorção do elemento pelas plantas. Embora responsivo ao Si, o eucalipto, à semelhança da maioria das dicotiledôneas (Marschner, 1995), não é uma planta acumuladora. Tomando-se como exemplo o maior valor de CSi medido nas plantas (14,66 mg vaso ${ }^{-1}$ de $\mathrm{Si}$ ), a conversão para teor de $\mathrm{SiO}_{2}$ na MSPA corresponde a $0,34 \mathrm{dag} \mathrm{kg}^{-1}$, o que, de acordo com esse mesmo autor, enquadra a espécie como planta não-acumuladora (teor de $\mathrm{SiO}_{2}$ na MSPA abaixo de $0,5 \mathrm{dag} \mathrm{kg}^{-1}$ ).

Ciênc. agrotec., Lavras. V.27, n.3, p.491-500, maio/jun., 2003 


\section{Latossolo}
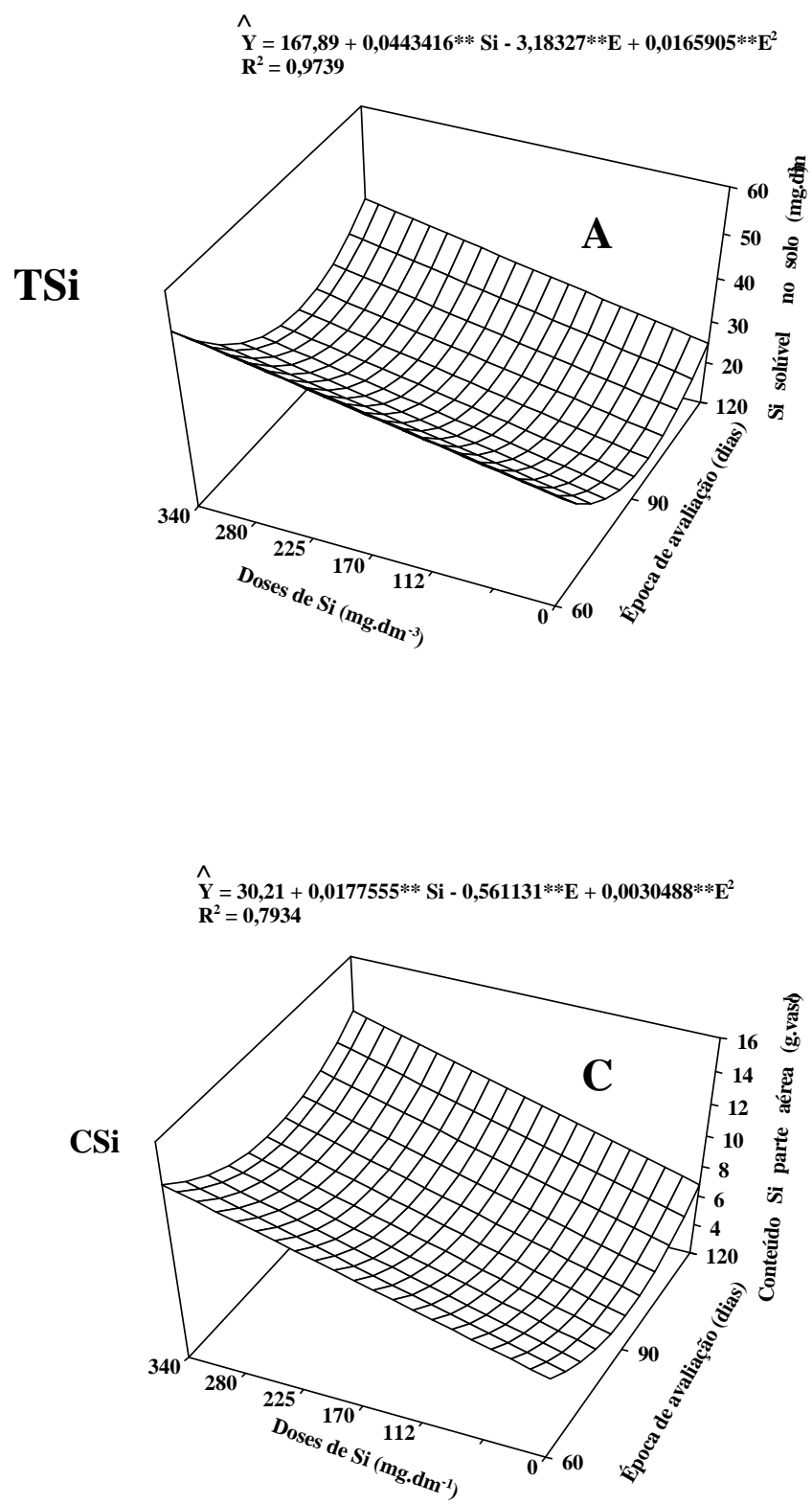

\section{Cambissolo}

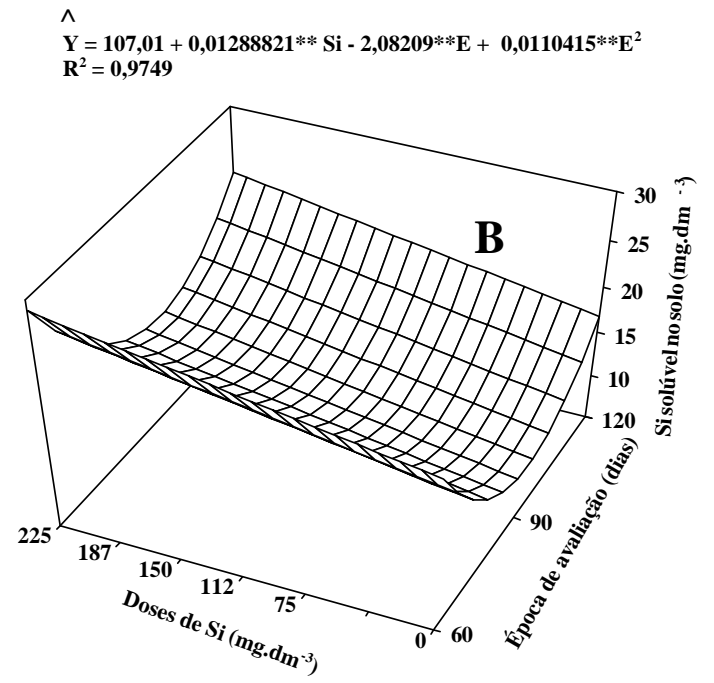

$\hat{\mathbf{Y}}=-\mathbf{5 8 , 7 0}+0,0188899 * * \mathrm{Si}+0,198975^{* *} \mathrm{E}-0,0010161^{* * *} \mathbf{E}^{2}$ $\mathbf{R}^{2}=0,7863$

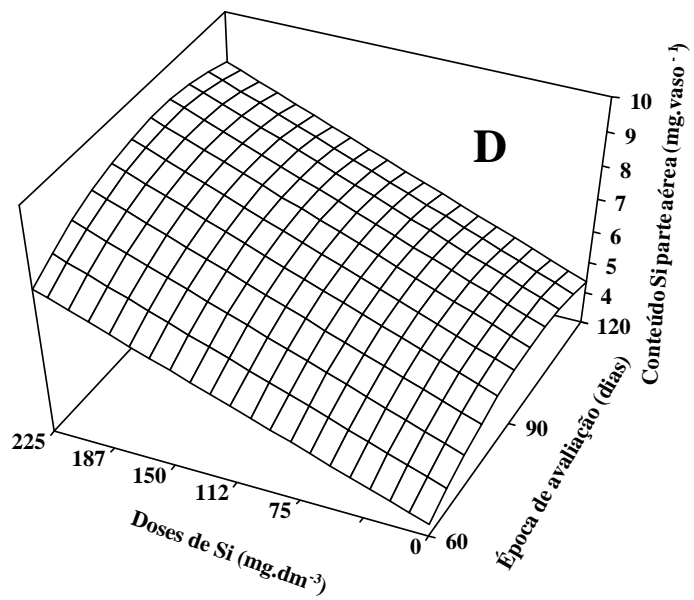

FIGURA 1 - Superfícies de resposta para Si solúvel em água (TSi) e conteúdo de Si (CSi) na MSPA de Eucalyptus grandis no Latossolo (A, C) e no Cambissolo (B, D), em função de doses de Si e épocas de avaliação. ** Coeficiente significativo a $1 \%$ pelo Teste $\mathrm{t}$. 
Eficiência de absorção e de utilização de silício pelas plantas

Nos dois solos e em cada época de avaliação, houve uma relação direta e positiva das doses de $\mathrm{Si}$ aplicadas com o acúmulo do elemento nas plantas e com a eficiência de absorção (Quadro 2). Entre épocas, com o avanço do período de crescimento do eucalipto, apesar do aumento da produção de matéria seca de raízes e do conteúdo de Si nas plantas, a eficiência de absorção diminuiu independentemente da dose do elemento.

Com esses resultados, inferem-se a existência de um mecanismo de exclusão de Si pelo eucalipto, à semelhança do que ocorre em duas outras dicotiledôneas: o tomate (Epstein, 1994) e o pepino (Marschner, 1995). Nesse contexto, parece que não se pode atribuir ao Si um papel metabólico capaz de influir no desenvolvi- mento normal da planta, o que pode ser comprovado analisando-se os dados de eficiência de utilização do elemento pela planta (Quadro 3).

No caso do Latossolo, em qualquer época de avaliação, houve relação inversa entre o incremento das doses de Si e a eficiência de utilização pela planta, ao passo que no Cambissolo ocorreu aumento na eficiência de utilização do Si em função das doses até os 90 dias, mas aos 120 dias, verificou-se uma tendência de nivelamento da eficiência em todas as doses (Quadro 3). Para uma mesma dose, embora a eficiência de utilização tenha aumentado bastante com o tempo, não se pode atribuir esse fato a uma contribuição metabólica do Si porque, em ambos os solos, a eficiência obtida no tratamento-controle, sem adição de $\mathrm{Si}$, praticamente não diferiu das demais doses, tendo, inclusive, superado os valores de eficiência de utilização observados nas doses mais elevadas.

QUADRO 2 - Eficiência de absorção de Si pelo eucalipto cultivado no Latossolo e no Cambissolo, em função das doses de Si e das épocas de avaliação.

\begin{tabular}{|c|c|c|c|c|c|c|c|c|c|}
\hline \multirow{3}{*}{$\begin{array}{l}\text { Dose Si } \\
\left(\mathrm{mg} \mathrm{dm}^{-3}\right)\end{array}$} & \multicolumn{9}{|c|}{ Época de avaliação (dias após o transplantio) } \\
\hline & \multicolumn{3}{|c|}{60} & \multicolumn{3}{|c|}{90} & \multicolumn{3}{|c|}{120} \\
\hline & $\begin{array}{c}\text { Acúm. Si } \\
\left(\text { mg vaso }^{-1}\right)\end{array}$ & $\begin{array}{l}\text { MS Raiz } \\
\quad(g)\end{array}$ & $\begin{array}{l}\text { Ef.Abs. } \\
\left(\mathrm{mg} \mathrm{g}^{-1}\right)\end{array}$ & $\begin{array}{l}\text { Acúm. Si } \\
\left(\mathrm{mg} \mathrm{vaso}^{-1}\right)\end{array}$ & $\begin{array}{l}\text { MS Raiz } \\
\quad \text { (g) }\end{array}$ & $\begin{array}{l}\text { Ef. Abs. } \\
\left(\mathrm{mg} \mathrm{g}^{-1}\right)\end{array}$ & $\begin{array}{l}\text { Acúm. Si } \\
\left(\mathrm{mg} \mathrm{vaso}^{-1}\right)\end{array}$ & $\begin{array}{c}\text { MS Raiz } \\
\text { (g) }\end{array}$ & $\begin{array}{l}\text { Ef. Abs. } \\
\left(\mathrm{mg} \mathrm{g}^{-1}\right)\end{array}$ \\
\hline & \multicolumn{9}{|c|}{..Latossolo... } \\
\hline 0 & 15,86 & 2,05 & 7,74 & 13,37 & 6,00 & 2,23 & 46,40 & 13,79 & 3,36 \\
\hline 112 & 14,38 & 1,59 & 9,04 & 20,75 & 6,79 & 3,05 & 54,55 & 15,91 & 3,43 \\
\hline 170 & 15,07 & 1,49 & 10,11 & 21,68 & 5,44 & 3,98 & 57,06 & 15,15 & 3,77 \\
\hline 225 & 19,65 & 1,89 & 10,40 & 26,70 & 6,56 & 4,07 & 60,25 & 15,16 & 3,97 \\
\hline 280 & 20,24 & 1,82 & 11,12 & 27,91 & 5,80 & 4,81 & 66,58 & 14,73 & 4,52 \\
\hline \multirow[t]{2}{*}{340} & 23,19 & 1,83 & 12,67 & 34,99 & 6,93 & 5,05 & 73,38 & 15,38 & 4,77 \\
\hline & \multicolumn{9}{|c|}{...Cambissolo... } \\
\hline 0 & 8,48 & 1,39 & 6,10 & 16,94 & 4,54 & 3,73 & 16,75 & 8,36 & 2,00 \\
\hline 75 & 8,47 & 1,16 & 7,30 & 18,82 & 3,76 & 5,00 & 19,62 & 8,92 & 2,20 \\
\hline 112 & 10,85 & 1,10 & 9,86 & 22,90 & 4,19 & 5,47 & 20,02 & 8,56 & 2,34 \\
\hline 150 & 12,57 & 0,89 & 14,12 & 24,78 & 4,24 & 5,84 & 27,64 & 10,68 & 2,59 \\
\hline 187 & 11,93 & 1,19 & 10,02 & 28,53 & 5,55 & 5,14 & 23,86 & 9,15 & 2,61 \\
\hline 225 & 14,55 & 1,16 & 12,54 & 30,72 & 4,51 & 6,81 & 28,76 & 9,72 & 2,96 \\
\hline
\end{tabular}


Portanto, as variações da eficiência de utilização de Si pelo eucalipto em função das doses do elemento e da época de avaliação parecem estar mais ligadas às características de crescimento (taxa de incremento de produção de matéria seca ao longo do período de condução do ensaio) inerentes à espécie.

\section{Translocação de silício nas plantas}

No Latossolo, na avaliação aos 60 dias, a relação de acúmulo de Si entre parte aérea e raízes (PA/R) au- mentou, de modo geral, seguindo o incremento das doses de $\mathrm{Si}$, ao passo que aos 90 dias, o valor dessa variável no tratamento-controle $(0,62)$ superou todos os demais. Aos 120 dias, praticamente não ocorreu diferença na relação de Si PA/R entre os tratamentos em função das doses. No Cambissolo, aos 60 e 90 dias após o transplantio, os valores de acúmulo de $\mathrm{Si} \mathrm{PA} / \mathrm{R}$ tenderam a acompanhar o incremento das doses, e tendência oposta foi verificada aos 120 dias, quando a relação de Si PA/R diminuiu com o aumento na dose do elemento (Quadro 4).

QUADRO 3 - Eficiência de utilização de Si pelo eucalipto cultivado no Latossolo e no Cambissolo, em função das doses de Si e das épocas de avaliação.

\begin{tabular}{|c|c|c|c|c|c|c|c|c|c|}
\hline \multirow{3}{*}{ 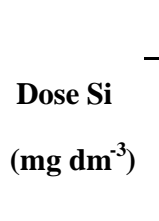 } & \multicolumn{9}{|c|}{ Época de avaliação (dias após o transplantio) } \\
\hline & \multicolumn{3}{|c|}{60} & \multicolumn{3}{|c|}{90} & \multicolumn{3}{|c|}{120} \\
\hline & $\begin{array}{l}\mathrm{MST}^{1} \\
(\mathrm{~g})\end{array}$ & $\begin{array}{l}\text { Acúm. Si } \\
\left(\text { (mg vaso }^{-1}\right)\end{array}$ & $\begin{array}{l}\text { Ef. Util. } \\
\left(\mathrm{g}^{2} \mathrm{mg}^{-1}\right)\end{array}$ & $\begin{array}{c}\text { MST } \\
\text { (g) }\end{array}$ & $\begin{array}{c}\text { Acúm. Si } \\
\left(\text { (mg vaso }^{-1}\right)\end{array}$ & $\begin{array}{l}\text { Ef. Util. }\left(\mathrm{g}^{2}\right. \\
\left.\mathrm{mg}^{-1}\right)\end{array}$ & $\begin{array}{l}\text { MST } \\
\text { (g) }\end{array}$ & $\begin{array}{l}\text { Acúm. Si } \\
\left(\mathbf{m g ~ v a s o - ~}^{-1}\right)\end{array}$ & $\begin{array}{l}\text { Ef. Util. } \\
\left(\mathrm{g}^{2} \mathbf{m g}^{-1}\right)\end{array}$ \\
\hline & \multicolumn{9}{|c|}{...Latossolo.. } \\
\hline 0 & 12,89 & 15,86 & 10,48 & 34,60 & 13,37 & 89,50 & 64,10 & 46,40 & 88,30 \\
\hline 112 & 9,52 & 14,38 & 6,30 & 41,08 & 20,75 & 81,33 & 69,19 & 54,55 & 87,76 \\
\hline 170 & 9,70 & 15,07 & 6,24 & 27,80 & 21,68 & 35,65 & 69,86 & 57,06 & 85,53 \\
\hline 225 & 11,78 & 19,65 & 7,17 & 36,32 & 26,70 & 49,41 & 73,21 & 60,25 & 88,96 \\
\hline 280 & 11,04 & 20,24 & 6,02 & 33,26 & 27,91 & 39,63 & 67,98 & 66,58 & 69,41 \\
\hline 340 & 11,14 & 23,19 & 5,35 & 40,93 & 34,99 & 47,88 & 71,67 & 73,38 & 70,00 \\
\hline & \multicolumn{9}{|c|}{ Cambissolo.. } \\
\hline 0 & 4,31 & 8,48 & 2,19 & 7,94 & 16,94 & 3,72 & 12,71 & 16,75 & 9,64 \\
\hline 75 & 4,24 & 8,47 & 2,12 & 8,25 & 18,82 & 3,62 & 14,18 & 19,62 & 10,25 \\
\hline 112 & 5,50 & 10,85 & 2,79 & 10,16 & 22,90 & 4,51 & 13,76 & 20,02 & 9,46 \\
\hline 150 & 5,95 & 12,57 & 2,82 & 9,90 & 24,78 & 3,95 & 17,45 & 27,64 & 11,02 \\
\hline 187 & 6,12 & 11,93 & 3,14 & 14,11 & 28,53 & 6,98 & 15,06 & 23,86 & 9,51 \\
\hline 225 & 9,28 & 14,55 & 5,92 & 13,00 & 30,72 & 5,50 & 16,47 & 28,76 & 9,43 \\
\hline
\end{tabular}

\footnotetext{
${ }^{1 /}$ Matéria seca total
} 
QUADRO 4 - Conteúdo de Si na matéria seca da parte aérea (SiPA) e das raízes (SiRA) e relação de acúmulo de Si parte aérea/raízes (PA/R) do eucalipto cultivado no Latossolo e no Cambissolo, em função das doses de Si e das épocas de avaliação.

\begin{tabular}{|c|c|c|c|c|c|c|c|c|c|}
\hline \multirow{3}{*}{$\begin{array}{l}\text { Dose } \mathrm{Si} \\
\left(\mathrm{mg} \mathrm{dm}^{-3}\right)\end{array}$} & \multicolumn{9}{|c|}{ Época de avaliação (dias após o transplantio) } \\
\hline & \multicolumn{3}{|c|}{60} & \multicolumn{3}{|c|}{90} & \multicolumn{3}{|c|}{120} \\
\hline & $\underset{\left(\text { mg vaso }^{-1}\right)}{\text { SiPA }}$ & $\begin{array}{l}\text { SiRA (mg } \\
\left.\text { vaso }^{-1}\right)\end{array}$ & $\mathbf{P A} / \mathbf{R}$ & $\underset{\left(\text { mg vaso }^{-1}\right)}{\text { SiPA }}$ & $\begin{array}{l}\text { SiRA (mg } \\
\left.\text { vaso }^{-1}\right)\end{array}$ & $\mathbf{P A} / \mathbf{R}$ & $\begin{array}{l}\text { SiPA }(\mathrm{mg} \\
\left.\text { vaso }^{-1}\right)\end{array}$ & $\begin{array}{l}\text { SiRA (mg } \\
\left.\text { vaso }^{-1}\right)\end{array}$ & $\mathbf{P A} / \mathbf{R}$ \\
\hline & & .... & ........ & ............... & Latossolo....... & ............ & . & 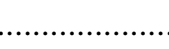 & .......... \\
\hline 0 & 8,49 & 7,37 & 1,15 & 5,11 & 8,26 & 0,62 & 7,61 & 38,79 & 0,20 \\
\hline 112 & 7,81 & 6,57 & 1,19 & 6,62 & 14,13 & 0,47 & 9,73 & 44,82 & 0,22 \\
\hline 170 & 9,11 & 5,96 & 1,53 & 5,71 & 15,97 & 0,36 & 9,35 & 47,71 & 0,19 \\
\hline 225 & 12,04 & 7,61 & 1,58 & 8,22 & 18,48 & 0,44 & 10,68 & 49,57 & 0,21 \\
\hline 280 & 12,32 & 7,92 & 1,55 & 8,66 & 19,25 & 0,45 & 11,21 & 55,37 & 0,20 \\
\hline 340 & 14,66 & 8,53 & 1,72 & 11,47 & 23,52 & 0,49 & 10,76 & 62,62 & 0,17 \\
\hline & & & & & ambissolo.. & & & & $\ldots \ldots \ldots$ \\
\hline 0 & 2,92 & 5,56 & 0,52 & 3,40 & 13,54 & 0,25 & 4,35 & 12,40 & 0,35 \\
\hline 75 & 3,08 & 5,39 & 0,57 & 4,49 & 14,33 & 0,31 & 5,26 & 14,36 & 0,37 \\
\hline 112 & 4,40 & 6,45 & 0,68 & 5,97 & 16,93 & 0,35 & 5,20 & 14,82 & 0,35 \\
\hline 150 & 5,06 & 7,51 & 0,67 & 5,66 & 19,12 & 0,30 & 6,77 & 20,87 & 0,32 \\
\hline 187 & 4,93 & 7,00 & 0,70 & 8,56 & 19,97 & 0,43 & 5,91 & 17,95 & 0,33 \\
\hline 225 & 8,12 & 6,43 & 1,26 & 8,49 & 22,23 & 0,38 & 6,75 & 22,01 & 0,31 \\
\hline
\end{tabular}

Em ambos os solos, principalmente no Latossolo, houve queda acentuada da relação de $\mathrm{Si}$ $\mathrm{PA} / \mathrm{R}$ entre as épocas, independentemente das doses fornecidas. No Cambissolo, a partir dos 90 dias, houve certa tendência de estabilização da partição de $\mathrm{Si}$ entre a parte áerea e raízes (Quadro 4).

Nos dois solos, admite-se que as plantas foram eficientes em absorver o Si fornecido e translocá-lo das raízes para a parte aérea até os 60 dias após o transplantio (fase inicial de crescimento). Fato similar foi observado no estádio inicial de desenvolvimento de plantas monocotiledôneas, como trigo e centeio (Jarvis, 1987), e das dicotiledôneas pepino (Miyake \& Takahashi, 1983) e soja (Miyake \& Takahashi, 1985), tendo havido redução no transporte de Si com o tempo.
Principalmente nas plantas cultivadas no Latossolo, houve retenção de Si nas raízes a partir dos 60 dias, semelhantemente ao que foi relatado para uma outra dicotiledônea, o tomateiro, que em qualquer estádio vegetativo acumula o elemento preferencialmente nas raízes (Okuda \& Takahashi, 1964). Nesse contexto, a retenção de $\mathrm{Si}$ pelo sistema radicular poderia constituir estratégia de resistência da planta ao déficit hídrico, conforme sugerido por Sangster (1978), já que, nesses períodos, o sistema radicular do eucalipto utilizaria a água necessária preferencialmente das moléculas de $\mathrm{H}_{4} \mathrm{SiO}_{4}$ resultantes da hidratação da sílica $\left(\mathrm{SiO}_{2}\right)$ depositada nas raízes.

Essa redução da eficiência de translocação de Si pelo eucalipto com o avançar da idade das plantas pode também ser constatada analisando-se o

Ciênc. agrotec., Lavras. V.27, n.3, p.491-500, maio/jun., 2003 
Quadro 5. No Latossolo, para todas as doses de $\mathrm{Si}$ testadas, houve uma queda de cerca de $50 \%$ na proporção de Si translocada, sucessivamente, a cada época de avaliação do ensaio. Os percentuais aumentaram com as doses apenas na primeira avaliação (60 dias), e já na segunda época (90 dias), a translocação observada na testemunha $(38,22 \%)$ superou os valores referentes às demais doses, não havendo, portanto, qualquer relação nítida entre as doses de $\mathrm{Si}$ e o percentual de translocação. Aos 120 dias, a diferença entre a testemunha e os tratamentos com as outras doses tamentos com as outras doses praticamente desapareceu.

No Cambissolo, ocorreu uma significativa redução nos percentuais de $\mathrm{Si}$ translocado da primeira para a segunda avaliação. Nesse solo, o efeito de doses sobre a translocação de $\mathrm{Si}$ foi menos pronunciado (90 dias) ou inexistente (120 dias) nas avaliações das plantas em estádio de desenvolvimento mais adiantado (Quadro 5). Tais observações reforçam a possível existência de algum mecanismo de controle do transporte de $\mathrm{Si}$ nas plantas de eucalipto.

QUADRO 5 - Translocação de Si em plantas de eucalipto cultivado no Latossolo e no Cambissolo, em função das doses de Si e das épocas de avaliação.

\begin{tabular}{|c|c|c|c|c|c|c|c|c|c|}
\hline \multirow{3}{*}{$\begin{array}{l}\text { Dose Si } \\
\left(\mathbf{m g ~ d m}^{-3}\right)\end{array}$} & \multicolumn{9}{|c|}{ Época de avaliação (dias após o transplantio) } \\
\hline & \multicolumn{3}{|c|}{60} & \multicolumn{3}{|c|}{90} & \multicolumn{3}{|c|}{120} \\
\hline & $\begin{array}{c}\text { Si planta } \\
\left.\text { (mg vaso }^{-1}\right)\end{array}$ & $\begin{array}{l}\text { SiPA } \\
\left.\text { vaso }^{-1}\right)\end{array}$ & $\begin{array}{c}\mathbf{S i} \\
\text { transl.( } \\
\%)\end{array}$ & $\begin{array}{c}\text { Si planta } \\
\left.\text { (mg vaso }^{-1}\right)\end{array}$ & $\begin{array}{l}\text { SiPA }(\mathbf{m g} \\
\left.\quad \text { vaso }^{-1}\right)\end{array}$ & $\begin{array}{c}\mathrm{Si} \\
\text { transl. } \\
(\%)\end{array}$ & $\begin{array}{c}\text { Si planta } \\
\left.\text { (mg vaso }^{-1}\right)\end{array}$ & $\begin{array}{l}\text { SiPA }(m g \\
\left.\text { vaso }^{-1}\right)\end{array}$ & 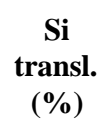 \\
\hline & \multicolumn{9}{|c|}{..Latossolo.. } \\
\hline 0 & 15,86 & 8,49 & 53,53 & 13,37 & 5,11 & 38,22 & 46,40 & 7,61 & 16,40 \\
\hline 112 & 14,38 & 7,81 & 54,31 & 20,75 & 6,62 & 31,90 & 54,55 & 9,73 & 17,84 \\
\hline 170 & 15,07 & 9,11 & 60,45 & 21,68 & 5,71 & 26,34 & 57,06 & 9,35 & 16,39 \\
\hline 225 & 19,65 & 12,04 & 61,27 & 26,70 & 8,22 & 30,79 & 60,25 & 10,68 & 17,73 \\
\hline 280 & 20,24 & 12,32 & 60,87 & 27,91 & 8,66 & 31,02 & 66,58 & 11,21 & 16,84 \\
\hline 340 & 23,19 & 14,66 & 63,22 & 34,99 & 11,47 & 32,78 & 73,38 & 10,76 & 14,66 \\
\hline \multicolumn{10}{|c|}{.Cambissolo... } \\
\hline 0 & 8,48 & 2,92 & 34,43 & 16,94 & 3,40 & 20,07 & 16,75 & 4,35 & 25,97 \\
\hline 75 & 8,47 & 3,08 & 36,36 & 18,82 & 4,49 & 23,86 & 19,62 & 5,26 & 26,81 \\
\hline 112 & 10,85 & 4,40 & 40,55 & 22,90 & 5,97 & 26,07 & 20,02 & 5,20 & 25,97 \\
\hline 150 & 12,57 & 5,06 & 40,25 & 24,78 & 5,66 & 22,84 & 27,64 & 6,77 & 24,49 \\
\hline 187 & 11,93 & 4,93 & 41,32 & 28,53 & 8,56 & 30,00 & 23,86 & 5,91 & 24,77 \\
\hline 225 & 14,55 & 8,12 & 55,81 & 30,72 & 8,49 & 27,64 & 28,76 & 6,75 & 23,47 \\
\hline
\end{tabular}




\section{CONCLUSÕES}

a) $\mathrm{Na}$ fase inicial de crescimento, as plantas de eucalipto absorvem muito pouco silício, sugerindo possuírem um mecanismo de exclusão do elemento;

b) A eficiência de translocação de Si é maior no estádio inicial de crescimento (60 dias), ficando a maior parte retida nas raízes a partir dessa época;

c) O Si solúvel em água no solo diminui com o tempo, situação não relacionada à absorção do elemento pelas mudas de eucalipto;

Si.

d) $\mathrm{O}$ eucalipto não é uma planta acumuladora de

\section{REFERÊNCIAS BIBLIOGRÁFICAS}

BELANGER, R. R.; BOWEN, P. A.; EHRET, D. L.; MENZIES, J. G. Soluble silicon, its role in the crop and disease management of greenhouse crops. Plant Disease, Quebec, v. 74, n. 4, p. 329-336, 1995.

CARVALHO, R.; FURTINI NETO, A. E.; CURI, N.; FERNANDES, L. A.; OLIVEIRA JUNIOR, A. C. Dessorção de fósforo por silício em solos cultivados com eucalipto. Revista Brasileira de Ciência do Solo, Campinas, v. 24, n. 1, p. 69-74, jan./mar. 2000.

CARVALHO, R.; FURTINI NETO, A. E.; SANTOS, C. D.; FERNANDES, L. A.; CURI, N.; RODRIGUES, D. C. Interações silício-fósforo em solos cultivados com eucalipto em casa-de-vegetação. Pesquisa Agropecuária Brasileira, Brasília, v. 36, n. 3, p. 557-565, mar. 2001.

EMADIAN, S. F.; NEWTON, R. J. Growth enhancement fo loblolly pine (Pinus taeda L.) seedlings by silicon. Journal of Plant Physiology, Stuttgart, v. 134, p. 98-103, 1989

EPSTEIN, E. The anomaly of silicon in plant biology. Proceedings Natural Academy Science, [S.1.], v. 91, p. 11-17, 1994.

FREIRE, J. C.; RIBEIRO, M. A. V.; BAHIA, V. G.; LOPES, A. S.; AQUINO, L. H. Resposta do milho cultivado em casa-de-vegetação a níveis de água em solos da região de Lavras (MG). Revista Brasileira de Ciência do Solo, Campinas, v. 4, n. 1, p. 5-8, 1980.

GALLO, J. R.; FURLANI, P. R. Determinação de silício em material vegetal pelo método colorimétrico do azul de molibdênio. Bragantia, Campinas, v. 37, n. 2, p. 5-11, 1978.
JARVIS, S. C. The uptake and transport of silicon by perennial ryegrass and wheat. Plant Soil, The Hague, v. 97, n. 3, p. 429-437, 1987.

JOLY, W. L. A química dos não metais. São Paulo: Edgard Blücher, 1966. 176 p.

JONES, L. H. P.; HANDRECK, K. A. Silica in soils, plants and animals. Advances in Agronomy, San Diego, v. 19, p. 107-147, 1967.

KORNDÖRFER, G. H.; DATNOFF, L. E. Adubação com silício: uma alternativa no controle de doenças de cana-de-açúcar e do arroz. Informações Agronômicas, Piracicaba, n. 70, p. 3, 1995.

MARSCHNER, H. Mineral nutrition of higher plants. 2. ed. London: Academic, 1995. 889 p.

McKEAGUE, J. A.; CLINE, M. G. Silica in soil solutions. Advances in Agronomy, San Diego, v. 15, n. 1, p. 339-396, 1963a.

McKEAGUE, J. A.; CLINE, M. G. Silica in soil solutions. I. The form and concentration of dissolved silica in aqueous extracts of some soils. Canadian Journal of Soil Science, Ottawa, v. 43, n. 1, p. 70-82, 1963 b.

MIYAKE, Y.; TAKAHASHI, E. Effect of silicon on the growth of solution-cultured cucumber plant. Soil Science and Plant Nutrition, Tokyo, v. 29, p. 71-83, 1983.

MIYAKE, Y.; TAKAHASHI, E. Effect of silicon on the growth of soybean plants in the solution culture. Soil Science and Plant Nutrition, Tokyo, v. 31, p. 625-636, 1985.

OKUDA, A.; TAKAHASHI, E. The role of silicon. The mineral nutrition of the rice plant. Proceedings Symposium International Rice Research Institute, [S.1.], p. 123-146, Feb. 1964.

RESENDE, M.; CURI, N.; REZENDE, S. B.; CORRÊA, G. F. Pedologia: base para distinção de ambientes. 2. ed. Viçosa: NEPUT, 1997. 367 p.

SANGSTER, A. G. Silicon in the roots of higher plants. American Journal of Botany, Columbus, v. 65, n. 9, p. 929-935, 1978.

SIDDIQI, M. Y.; GLASS, A. D. M. Utilization index: a modified aproach to the estimation and comparison of nutrient utilization efficiency in plants. Journal of Plant Nutrition, Monticello, v. 4, p. 289-302, 1981. 